\title{
Atomic structure of dislocation kinks in silicon
}

\author{
R.W. Nunes ${ }^{1}$, J. Bennetto ${ }^{2}$, and David Vanderbilt ${ }^{2}$ \\ ${ }^{1}$ Complex System Theory Branch, Naval Research Laboratory, Washington DC, 20375-53459 \\ and Computational Sciences and Informatics, George Mason University, Fairfax, Virginia \\ ${ }^{2}$ Department of Physics and Astronomy, Rutgers University, Piscataway, New Jersey 08855-0849
}

(July 15, 1997)

\begin{abstract}
We investigate the physics of the core reconstruction and associated structural excitations (reconstruction defects and kinks) of dislocations in silicon, using a linear-scaling density-matrix technique. The two predominant dislocations (the $90^{\circ}$ and $30^{\circ}$ partials) are examined, focusing for the $90^{\circ}$ case on the single-period core reconstruction. In both cases, we observe strongly reconstructed bonds at the dislocation cores, as suggested in previous studies. As a consequence, relatively low formation energies and high migration barriers are generally associated with reconstructed (dangling-bondfree) kinks. Complexes formed of a kink plus a reconstruction defect are found to be strongly bound in the $30^{\circ}$ partial, while the opposite is true in the case of $90^{\circ}$ partial, where such complexes are found to be only marginally stable at zero temperature with very low dissociation barriers. For the $30^{\circ}$ partial, our calculated formation energies and migration barriers of kinks are seen to compare favorably with experiment. Our results for the kink energies on the $90^{\circ}$ partial are consistent with a recently proposed alternative double-period structure for the core of this dislocation.
\end{abstract}

61.72.Lk, 71.15.Pd, 71.15.Fv

\section{INTRODUCTION}

Dislocations are of fundamental importance in the physics of semiconductors, both from a mechanical and from an electronic point of view. They are the carriers of plasticity in crystals and act as trapping and scattering centers for electronic carriers. A wealth of experimental information is available about the properties of dislocations in tetrahedrally bonded semiconductors $1 \mathrm{O} \mathrm{In} \mathrm{Si}$, the predominant slip systems are the $60^{\circ}$ and the screw dislocations oriented along [110] directions in a $\{111\}$ slip plane. Both are known to occur in the glide configuration and to dissociate into pairs of partial dislpcations bounding a ribbon of intrinsic stacking fault. 13 Dissociation lowers the strain energy and is made energetically favorable by the low energy of the stacking fault in this material. (Evidence indicates that the above is also true in the case of germanium and for III-V and II-VI semiconductors 1, 2) The resulting $90^{\circ}$ and $30^{\circ}$ partials are believed to undergo core reconstruction, which eliminates the unsaturated bonds, thus restoring the fourfold coordination of the atoms at the cores. This picture is consistent with the low density of dangling bonds, as suggested by EPR measurements.

Dislocation motion occurs by nucleation and propagation of kinks along the dislocation line. Due to thermal fluctuations or the action of an applied stress, double kinks can be nucleated along the dislocation line. When these reach a critical separation, dissociation occurs and the individual kinks propagate in opposite directions thus generating a displacement of a dislocation segment. A detailed understanding of the atomic-scale structure of the kinks and the barriers associated with their motion is thus of the greatest importance.
In semiconductors, according to the theoretical model proposed by Hirth and Lothe (HL), 3 double-kink nucleation and the high Peierls potential barrier to kink motion control the rate of dislocation propagation. This is to be contrasted with the case of metals, where kinks experience a very low barrier to motion, and the rate is controlled by nucleation only. The HL model is often used in the interpretation of dislocation mobility experiments, although the occurrence of such high Peierls barriers has been questioned by some authors.9 Furthermore, an alternative theoretical model has been proposed in which dislocation motion is controlled by the pinning of kinks by obstacles distributed along the dislocation line.10, 11 Recent experimental evidence suggests that the barriers are indeed high, but experiments capnet clearly decide between these two theoretical models. -1 A complete microscopic picture is still lacking. Related issues, such as the dependence of dislocation mobility on doping and the photoplastic effect in semiconductors 1 would also profit from a better understanding of dislocation structure at the atomic level.

On the computational side, large-scale problems of this nature have been mostly studied by using classical interatomic potentials. Such studies are not always reliable, since these potentials are often unable to reproduce effects of intrinsic quantum-mechanical nature such as bond reconstruction and Peierls or Jahn-Teller symmetry breaking. For example, while the Stillinger-Weber 22 potential has been used to study the core reconstruction and kinks of the $30^{\circ}$ partial, 13 it fails to reproduce the spontaneous symmetry-breaking core reconstruction of the $90^{\circ}$ partial from the symmetric "quasi-fivefold" reconstruction.14.15 A proper quantum-mechanical description of the electronic structure is clearly needed. 
One is thus led to consider tight-binding (TB) and $a b$ initio methods.

Recent $a b$ initio and TB theoretical works have concentrated on such issues as the core reconstruction of the $90^{\circ}$ partial, 14,16 and the elastic interaction between dislocations of a dipole in the shufflet-7 and glide sets. 16 Using a relatively small supercell, one first-principles study has determined a kink mobility barrier in the $30^{\circ}$ partial, 18 but only one kink species was studied, out of a very rich variety characteristic of this system. As will become clear from the conclusions of the present work and from Ref. 13, the formation and migration energies of other kinks are needed for a proper comparison with the experimental results. An important recent development is our prediction, 19 on the basis of classical-potential, tightbinding, and $a b$ initio calculations, that the reconstruction of the $90^{\circ}$ partial in $\mathrm{Si}$ is not the above-mentioned symmetry-breaking structure, as had generally been accepted in the theoretical literature.14 40,20 27 Instead, we proposed a double-period (DP) reconstruction whose core structure is reminiscent of that of a double-kink of minimal length that would form on the core of the original single-period (SP) structure 19 Cluster calculations on kinks and soliton 28 in the SP recpenstruction of the $90^{\circ}$ partial have also been reported 232522 These calculations have identified many of the basic types of defects in this system, but must be taken at a semi-quantitative level, since they suffer from the lack of coupling of the defect local strain fields with the lattice elastic fields.

To address properly the issues related to dislocation mobility, a comprehensive study of dislocation kink structure and dynamics would require the use of very large supercells, for which the application of ab initio techniques is still computationally prohibitive. In view of this, the natural choice is the application of more efficient quantum-mechanics based methods to study the electronic and structural excitations in the dislocation cores. In this work, we employ the tight-binding-total-epergy (TBTE) Hamiltonian of Kwon and collaborators 29 to carry out a detailed atomistic study of the atomic structure of both the $30^{\circ}$ and the $90^{\circ}$ partial dislocations in $\mathrm{Si}$. To make these calculations tractable, we use the linear-scaling or "O$(N)$ " method of Li, Nunes and Vanderbilt 30 to solve for the electronic-structure contribution to energies and forces, enabling us to treat system sizes up to $10^{3}$ atoms easily on a workstation platform. Our work addresses some of the fundamental issues associated with these two systems. More specifically, we address the ground-state structural properties of the dislocation cores and of defects in the core, such as kinks and reconstruction defects (RD), as well as energy barriers to motion of the various defects.

In this work, when considering the $90^{\circ}$ partial, we will discuss only the SP reconstruction. Despite the fact that this is not the correct ground state for this dislocation in $\mathrm{Si}$, we hope that understanding this somewhat simpler system will help us in the study of the myriad of defects in the more complicated ground-state DP reconstruction, to which the former is related 19 Moreover, we should keep in mind that the $90^{\circ}$ partial is equally important in other materials, such as germanium $(\mathrm{Ge}$ diamond $(\mathrm{C})$, and the III-Vand II-VI semiconductors. 13 Preliminary calculations, 19 using a Keating model,31 indicate that in $\mathrm{C}$ the SP reconstruction is more likely to be lower in energy, while $\mathrm{Ge}$, like $\mathrm{Si}$, would prefer the DP structure. More accurate calculations are needed to reveal which of the two reconstructions would be favored in each case. Therefore, the study of the SP structure is still important from a theoretical point of view.

The paper is organized as follows. The next section contains the technical details of the calculations we performed. In Sec. III, we discuss our results for the core reconstruction and related defects in the $30^{\circ}$ partial dislocation. Our main results for the SP reconstruction of the $90^{\circ}$ partial are described in Sec. IV. Finally, in Sec. V we summarize the main conclusions and results, and compare our kink energies and barriers with the available experimental results. In particular, we will argue that our results appear to be consistent with the HL theory of dislocation glide.

\section{TECHNICAL DETAILS OF THE CALCULATIONS}

We use the TBTE parameters of Kwon et al.29 which describe well the acoustic phonon modes and elastic constants of $\mathrm{Si}$, thus being adequate to describe the strain fields associated with the dislocation cores and related defects. Owing to its good transferability between different crystal structures, ranging from diamond to FCC, this Hamiltonian is also expected to give a good description of the coordination defects in the present study. The $\mathcal{O}(N)$ method of Li et al.30 is used to solve for the electronic structure contribution to the energies and forces. For the density matrix, we initially work at a real-space cutoff $R_{c}=6.2 \AA$ on the range of the density matrix used in the tests presented in Ref. 30 for the $\mathcal{O}(N)$ method. In a second stage, we improve the convergence of our results by further relaxing the ionic positions and the electronic structure with a larger cutoff value of $R_{c}=7.3 \AA$. The numerical minimization of the $\mathcal{O}(N)$ functional was carried out by the conjugate-gradient algorithm, with the internal line minimization performed exactly. To obtain the right number of electrons, the chemical potential is adjusted iteratively, in each case. Usually, this procedure has to be repeated only at the initial steps of the structural relaxation procedure, after which the chemical potential converges to the adequate value and remains constant. Ground-state structures were computed by allowing all atomic coordinates to relax fully (average forces less than $0.1 \mathrm{meV} / \AA$ ).

Our supercells are chosen with the dislocation direction (corresponding to a $[1 \overline{1} 0]$ crystalline direction) lying on the $y$-axis. The glide plane (which contains a stacking 
fault) is normal to the [111] direction and coincides with the $x y$ plane of the cell. (Fig. 1 shows the glide plane of the $30^{\circ}$ partial dislocation, with the crystalline directions indicated.) The $z$ direction of the cell is thus parallel to the [111] direction. Each supercell contains two dislocations having opposite Burgers vectors (a dislocation dipole), which allows us to use periodic boundary conditions. Supercell vectors are chosen such as to array the dislocations in a quadrupole configuration, as suggested in Ref. 14, to avoid the spurious shear strains associated with the minimal dipole cell.

To ensure the convergence of our calculations with respect to supercell size, we used three different cells, containing 216, 264, and 308 atoms respectively, for the simulation of the reconstructed core of the $30^{\circ}$ partial dislocation. Each cell corresponds to a slab of atoms at a $60^{\circ}$ angle with respect to the dislocation direction, including twice the lattice period in that direction, to allow for the period-doubling reconstruction of the $30^{\circ}$ partial. The two parameters characterizing the geometry of each cell are the separation between the two dislocations in the glide plane (i.e., the width of the stacking fault) within a given unit cell, and the distance between the periodicimage dipoles along the $z$ direction. In our calculations, these distances are, respectively, $15.0 \AA$ and $18.8 \AA$ for the 216-atom cell, $18.3 \AA$ and $18.8 \AA$ for the 264 -atom cell, and $18.3 \AA$ and $21.9 \AA$ for the 308 -atom cell.

The supercells for the computation of defect energies are obtained by repeating the core slabs several times along the dislocation direction. The defect energies we quote are referred to the corresponding supercell containing defect-free (but reconstructed and fully relaxed) dislocations. For the kinks in the $30^{\circ}$ partial, each of the core slabs were repeated three times (two and a half times for the RD, and three and a half times for the kink$\mathrm{RD}$ complexes) along the dislocation direction, so that the defect-defect separation along the line was $19.2 \AA$ or larger, depending on the type of defect. Below, we describe the procedure we used for the computation of defect energy barriers. Because of the higher computational demands involved, in this case we employed only the smaller cells (three times the 216-atom slab for kinks and two and a half times the same slab for the RD).

Table I in the next section illustrates the convergence of our results with respect to dislocation separation. As a further check, we also computed the energies of the core and of one the kinks (the LK kink, as described below) with an even larger slab, consisting of 600 atoms for the reconstructed core (1800 atoms for the defect). In this case, dislocation distances are $24.9 \AA$ in the $x y$ plane and $31.4 \AA$ in the $z$ direction. The change in defect energy with respect to the 308-atom slab was only $\sim 0.02 \mathrm{eV}$. To test the effect of defect interaction, this kink was studied with a larger kink-kink separation (with the smallest slab repeated eight times), which produced a change of only $\sim 0.01 \mathrm{eV}$ in the energy. Therefore, we consider our calculations to be converged within $0.03 \mathrm{eV}$ with respect to core-core and defect-defect interactions. To estimate the error involved our choice of cutoff for the density matrix, kink and core energies were computed using a larger cutoff $\left(R_{c}=8.1 \AA\right)$. The kink formation energy changed by only $\sim 0.06 \mathrm{eV}$, which justifies our choice of cutoff. From these results we can also estimate that our defect energy barriers are converged within $0.3 \mathrm{eV}$. The supercells used for the study of the $90^{\circ}$ partial, are as described in Refs. 15,19. In this case, despite the fact that we are only interested in the qualitative nature of our results, our values are well converged, with dislocation distances on the order of $26.6 \AA$, and defect-defect separations of at least $\sim 13.4 \AA$. (As in the case of the $30^{\circ}$ partial, barriers are computed using smaller cell sizes, corresponding to a dislocation separation of $13.3 \AA$.)

Barrier energies were calculated by identifying the $3 \mathrm{~N}$ dimensional configuration-space vector $\mathbf{R}_{12}=\mathbf{R}_{2}-\mathbf{R}_{1}$ pointing from one equilibrium position $\mathbf{R}_{1}$ of the defect to a neighboring position $\mathbf{R}_{2}$, and defining a reaction coordinate $Q=\mathbf{R} \cdot \mathbf{R}_{12}$ measuring the progress from $\mathbf{R}_{1}$ to $\mathbf{R}_{2}$. Then, for a series of values of this coordinate, we computed the energy with this coordinate fixed and all others fully relaxed. This approach is efficient in simple cases, but we find that it often fails to converge to the saddle-point configuration when the reaction path 32 makes sharp angles with respect to $\mathbf{R}_{12}$. In these cases, we can usually find two configurations, $\mathbf{R}_{\mathbf{1}}^{\prime}$ and $\mathbf{R}_{\mathbf{2}}^{\prime}$, near the saddle-point, with nearly the same value of $Q$ but with opposite forces along $\mathbf{R}_{12}$. By exploring the space spanned by $\mathbf{R}_{12}$ and $\left(\mathbf{R}_{\mathbf{2}}^{\prime}-\mathbf{R}_{\mathbf{1}}^{\prime}\right)$ while allowing all other coordinates to relax, we were able to determine the energy barriers with good accuracy for all cases studied (average forces less than $1.0 \mathrm{meV} / \AA$ ).

\section{THE $30^{\circ}$ PARTIAL DISLOCATION}

\section{A. Core reconstruction}

In Fig. 11(a), a top view of the atomic structure of the unreconstructed $30^{\circ}$ partial in the glide plane is shown. The shaded area represents the stacking fault, and the dislocation line is indicated by the boundary between shaded and unshaded areas. The crystalline directions are also displayed. Atoms shown as white (black) are above (below) the glide plane; each atom is bonded to another either above or below it, and these are not shown in the picture. Thus, fourfold coordinate atoms have three of their bonds in the plane of the figure. The atoms at the core of the defect are threefold coordinated, with a dangling bond lying nearly parallel to the dislocation line. In Fig. 1 (b) we show a reconstruction in which the fourfold coordination of the atoms at the core is restored by atoms bonding in pairs along the line, leading to a doubling of the period in that direction. This reconstruction is well accepted as being the ground-state of the $30^{\circ}$ partial and has been discussed theoretically by other authors.21 33 34. 33 In Ref 13 , it was found to be $0.21 \mathrm{eV} / \AA$ 
(a)

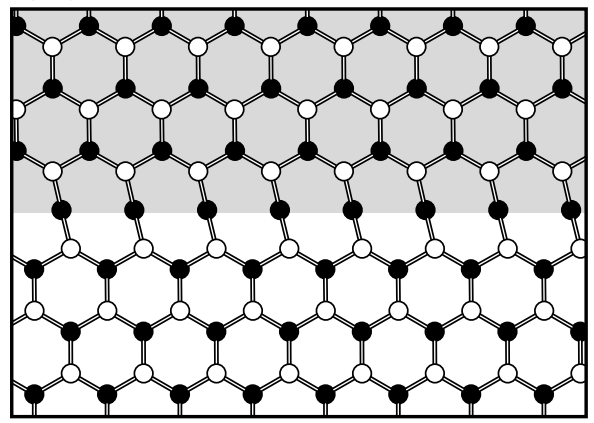

(b)

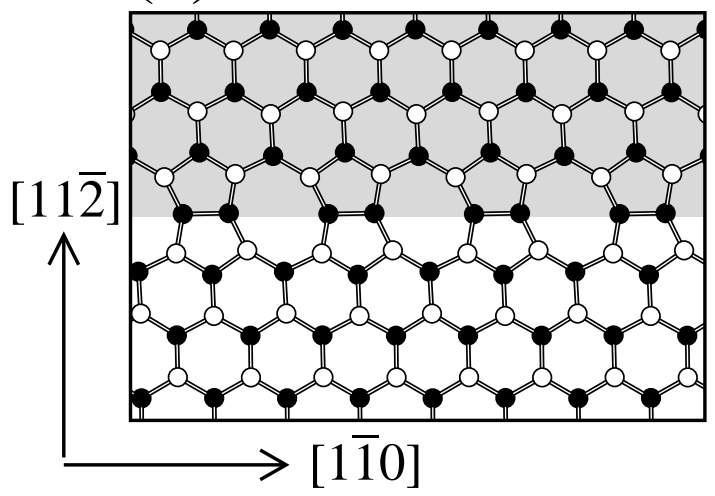

FIG. 1. (a) Unreconstructed core of the $30^{\circ}$ partial dislocation, viewed from above the (111) slip plane. Shaded region indicates stacking fault. Black (white) atoms lie below (above) the slip plane. (b) Same view of the double-period reconstructed structure. Crystalline directions are also shown.
TABLE I. Formation energy of defects in the $30^{\circ}$ partial dislocation, in eV. Defect energies are referred to a defect-free dislocation core. TB results for three supercell sizes are shown. For the PSD, supercells contain $5 / 6$ of the number of atoms shown. For the LK, in the third column we indicate in parenthesis the formation energy computed with a 1800-atom cell. Fourth column contains Keating energy computed for the largest cell (with "Keating $+0.4 \mathrm{eV}$ " numbers in parenthesis).

\begin{tabular}{lcccc}
\hline \hline & 648 atoms & 792 atoms & 924 atoms & Keating \\
\hline PSD & 1.35 & 1.32 & 1.33 & \\
LK & 0.52 & 0.37 & $0.35(0.33)$ & $-0.06(0.34)$ \\
LK $^{\prime}$ & 0.97 & 0.81 & 0.76 & $0.44(0.84)$ \\
RK & 0.93 & 1.20 & 1.24 & $1.00(1.40)$ \\
RK $^{\prime}$ & 1.64 & 1.84 & 1.85 & $1.30(1.70)$ \\
\hline \hline
\end{tabular}

lower in energy than the unreconstructed structure, using a Stillinger-Weber potential. We find a higher value of $0.36 \mathrm{eV} / \AA$ for the reconstruction energy.

A look at the distribution of bond lengths for this structure shows that the reconstruction is indeed strong, with maximum bond-length deviations of only $3.0 \%$ (maximum and minimum bond lengths are $2.423 \AA$ and $2.308 \AA$, respectively) with respect to $\mathrm{Si}$ bulk values $(2.351 \AA)$. The core energy is mostly due to the strain associated with bond-angle distortions at the core of the defect, with bond angles ranging between $\sim 90^{\circ}$ and $\sim 126^{\circ}$ $\left(109.5^{\circ}\right.$ is the bulk value). No mid-gap levels are expected for this structure, in accordance with the EPR evidence.t 1 -

A rich variety of core defects is associated with this reconstruction, including kinks and RDs, and complexes of these basic types. A very extensive study of these defects is found in Ref. 13 , including structural features and energetics under a Stillinger-Weber potential. To a large extent, our study of this specific dislocation relies on this previous study, adding to it the benefits of a quantummechanical treatment of the electronic structure. More specifically, the defects considered in this work are the ones identified in Ref. 13. As we proceed, it will be seen that some of our results differ qualitatively from those in Ref. 13, and also that we find a better agreement with the experimental results.

\section{B. Phase switching defect (PSD)}

Fig. 2(a) shows a RD associated with the core of the $30^{\circ}$ partial. We shall refer to this defect as a phase switching defect (PSD) 28 The existence of such defects has been hinted at since the realization that the core of the partials might undergo reconstruction. 1.23 We computed the energy of a fully relaxed PSD by repeating the atom slabs five times along the $\langle 110\rangle$ direction, and introducing one PSD in each dislocation line. Our value for the PSD formation energy is $1.32 \mathrm{eV}$ (see Table I be- 
(a)

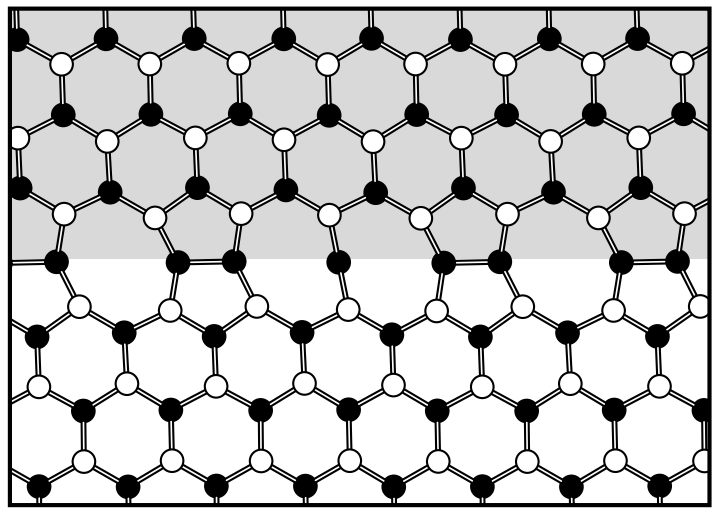

(b)

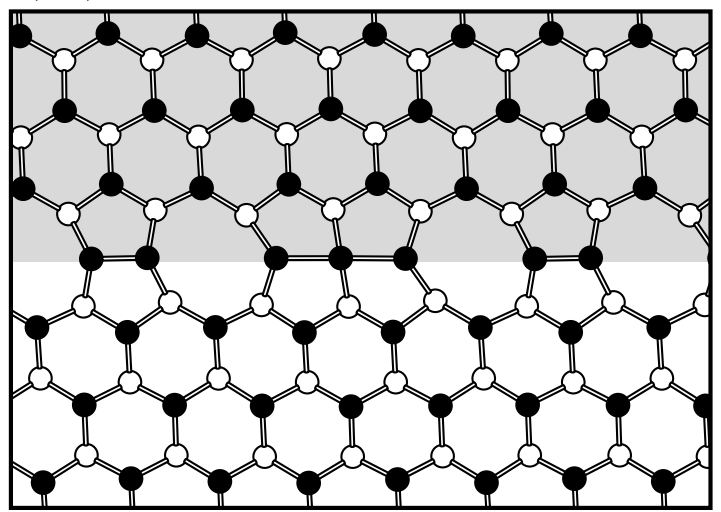

FIG. 2. (a) Core structure of a phase-switching defect (PSD), which is a reconstruction defect in the core 30-partial dislocation. The phase of the reconstructed bond along the dislocation line is switched, going through the defect. (b) Saddle-point configuration for the propagation of a PSD along the core.

low), which is somewhat higher than the value of 0.81 $\mathrm{eV}$ obtained in Ref. 13. We believe our result to be more reliable, given the quantum-mechanical nature of our approach, in particular for a defect containing a dangling bond. To a first approximation this defect can be understood as a $p$ dangling-bond defect, which indicates that a formation energy on the order of $1 \mathrm{eV}$ (roughly the bond-breaking energy in bulk $\mathrm{Si}$ ) is to be expected. The exact value is determined by the relaxation of the atoms surrounding the defect.

We also computed the migration barrier for the propagation of the PSD along the dislocation direction. The relaxed structure of the barrier (saddle-point) configuration is shown in Fig. 2(b). In this case, the symmetry between adjacent positions of the defect along the line indicates that the saddle-point configuration is at the halfway position. It was somewhat surprising to find that even in this case, we had to resort to a two-dimensional reaction coordinate as described Sec. II Our saddle-point configuration, with an energy barrier of $0.3 \mathrm{eV}$, is very similar to that in Ref. 13. In can be seen that the atom at the center becomes fivefold coordinated, which leads to a smooth process of bond substitution as the PSD propagates to the right. This explains the low energy barrier involved in this process.

\section{Kinks}

The period doubling of the reconstructed core gives rise to a multiplicity of kinks in this system. Two distinct families of such defects appear, depending on whether the dislocation "kinks" to the left (Fig. 3) or to the right (Fig. 4). The period doubling of the core introduces a choice of phase of the core reconstruction both ahead of, and behind, the kink. Of the four configurations generated in this way, two of them (those necessarily containing a coordination defect) will be classified as PSD-kink complexes, and will be considered in Sec. IIID. The remaining two configurations will be classified as "pure" kinks and are considered here. The two left kinks LK and $L K^{\prime}$ are shown in Figs. 3(a-b), while the two right kinks RK and $\mathrm{RK}^{\prime}$ are shown in Fig. $4(\mathrm{a}-\mathrm{b})$.

The energies for each type of kink were computed using the TBTE Hamiltonian, as well as with a classical Keating model, 31 with the parameters proposed in Ref. 35, in order to look at the local-strain contributions to the energy of each defect. In Table I, we show the TBTE results for each of the three slabs described in section II, along with the Keating results for the 924-atom cell. For one of the kinks, the energy computed with a 1800-atom slab is also shown in parenthesis; note the convergence of these results with respect to cell size. Next, we discuss the results for each kink family in more detail.

\section{Left kinks}

The left kinks LK and LK' are shown in Fig. 3, together with the saddle-point configuration for the LK $\rightarrow$ $\mathrm{LK}^{\prime}$ translational motion. The energies, as given in Table I, show that reconstruction produces low energy kinks in this case, as compared to the energy of the unreconstructed PSD defect. At first sight, the formation energy for these reconstructed defects is expected to be mostly associated with the local strain at the kink cores. The Keating-model results can give us an estimate of these local-strain effects. The LK is found to add no additional strain on that imposed by the core reconstruction itself, as can be seen by its slightly negative energy. On the other hand, the $\mathrm{LK}^{\prime}$ kink is found to have a Keating formation energy of $0.44 \mathrm{eV}$. It is interesting to note that the relative Keating energy of the two left kinks is in good agreement with the TB results. This is actually true for all four kink types, as can be seen be comparing differences of the Keating energies in the fourth column 
(a)

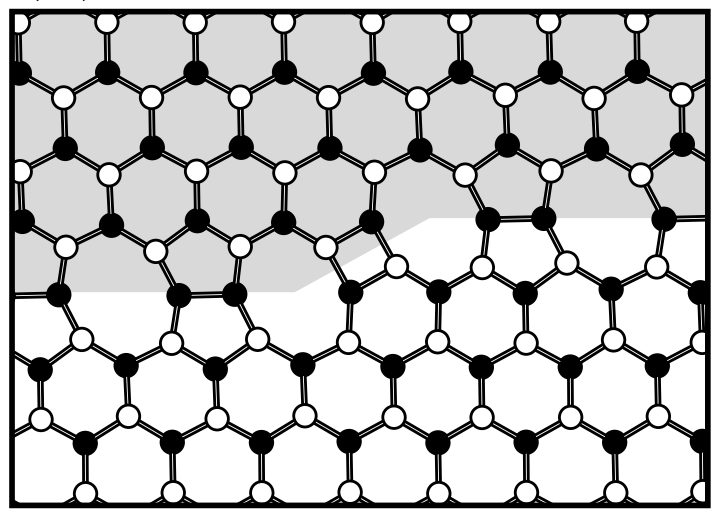

(b)

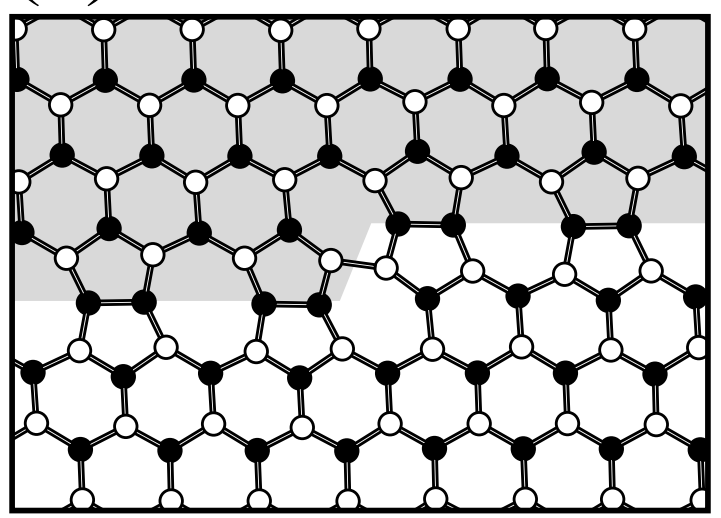

(c)

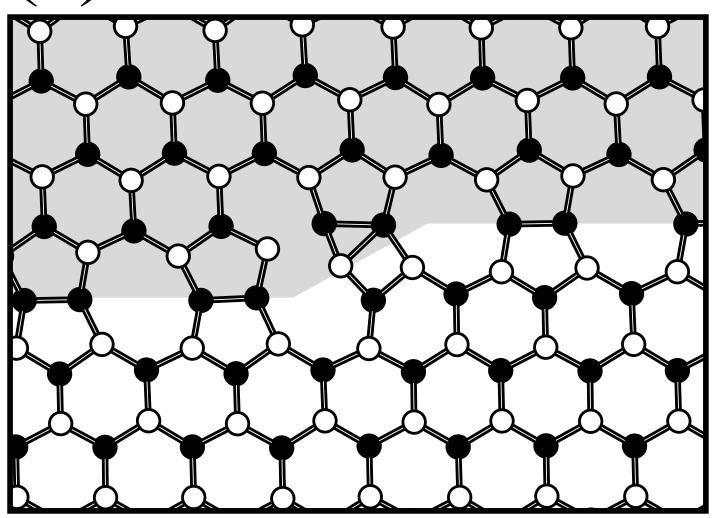

FIG. 3. Core structure of the left kinks in the $30^{\circ}$ partial, and associated transition state. Kink notation is explained in the text. (a) LK kink. (b) LK' kink. (c) Transition state for the $\mathrm{LK} \rightarrow \mathrm{LK}^{\prime}$ transformation. (a)

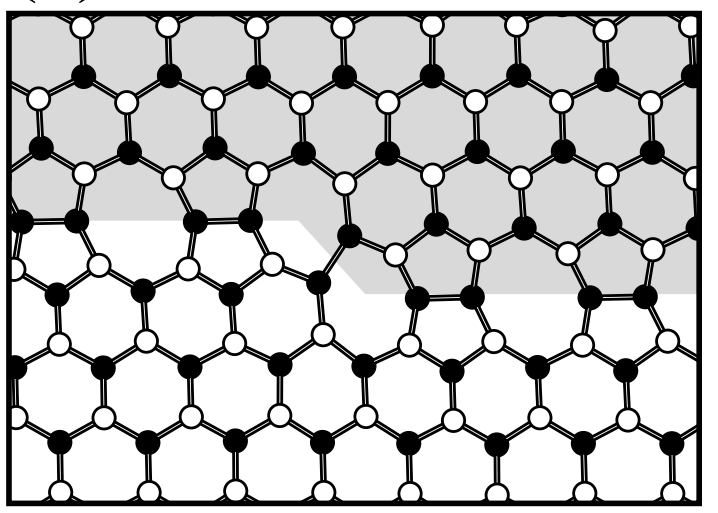

(b)

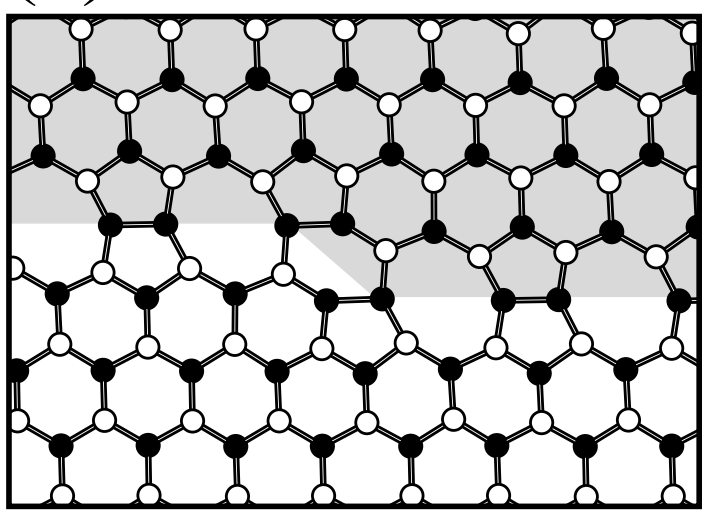

(c)

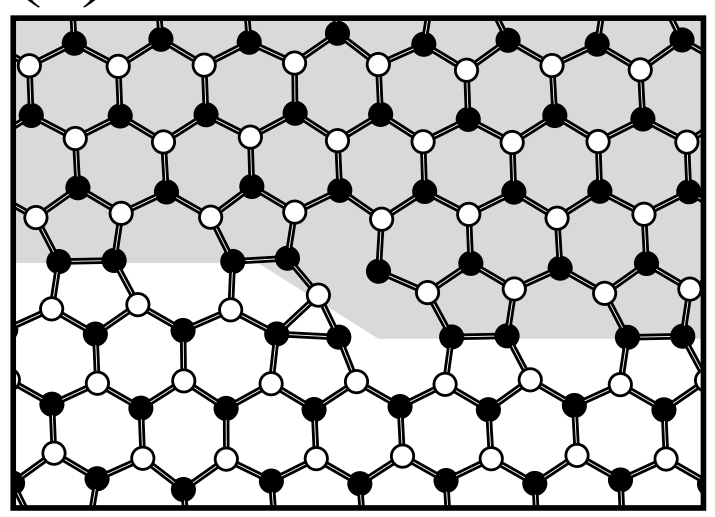

FIG. 4. Core structure and transition state of right kinks in the $30^{\circ}$ partial. Kink notation is explained in the text. (a) RK kink. (b) RK' kink. (c) Transition state for the RK $\rightarrow$ $\mathrm{RK}^{\prime}$ transformation. 
of Table I. In the last column, we add a constant shift of $0.4 \mathrm{eV}$ to each Keating energy. It is evident that the "Keating + $0.4 \mathrm{eV}$ " results are reasonably close to the TB ones. In view of this, we conclude that a roughly constant band-structure energy of $0.4 \mathrm{eV}$ is associated with each kink.

For the saddle-point configuration in Fig. 3 (c) we computed an energy barrier of $1.52 \mathrm{eV}$. This result is in very good agreement with experimental estimates. In our concluding section, we will discuss more extensively the significance of our results in light of the available experimental evidence. Here, we note that such a high barrier can be understood by the presence of severe bond-bending and stretching distortions at the core of the defect, along with the presence of malcoordinated atoms. Bond angles as small as $50.4^{\circ}$ are found, as well as bonds stretching to $2.80 \AA$.

\section{Right kinks}

Shown in Fig. 1 are the two kinks of the right family, RK and RK', together with the saddle-point corresponding to the $\mathrm{RK} \rightarrow \mathrm{RK}^{\prime}$ reaction. Despite the fact that both kinks are fully reconstructed, the formation energies of $1.24 \mathrm{eV}$ for RK and $1.85 \mathrm{eV}$ for $\mathrm{RK}^{\prime}$ are surprisingly high. Again, note the agreement between the Keating values and the TB ones, after adding a constant shift of $0.4 \mathrm{eV}$ to the former. No single structural feature of the right kinks could be traced in order to explain the unexpected formation energies. The minimum and maximum distortions of bond lengths and angles do not vary drastically among the four kink types.

To help us better understand these results, we observe that the Keating energies can be decomposed in an atomby-atom basis. Bond bending energies, associated with changes in the angle between two bonds, are assigned to the vertex atom, and half of the bond stretching energy of a given bond is assigned to each of the two participating atoms. To examine the nature of the strain fields associated with each kink type, using our largest cells (924 atoms), we looked at these atomic energies integrated over shells of atoms defined by their distance from the core of the defect. (Since our supercells contain two cores and thus two defects, we always choose the shortest distance to a defect.) The integrated energies are then defined by

$$
E^{d}(R)=\sum_{R_{i} \leq R} E_{i}^{d}\left(R_{i}\right)-E_{i}^{c}\left(R_{i}\right)
$$

where the Keating energy $E_{i}^{c}\left(R_{i}\right)$ of each atom in a correspondent kink-free supercell (containing only the dislocation dipole) is subtracted, and we sum over all atoms within a distance $R$ from the kink. The results are shown in Fig. 5. We see that the kink energies are determined by the medium-range behavior of the associated strains. At short range $(R<3.0 \AA)$ the LK kink is actually the

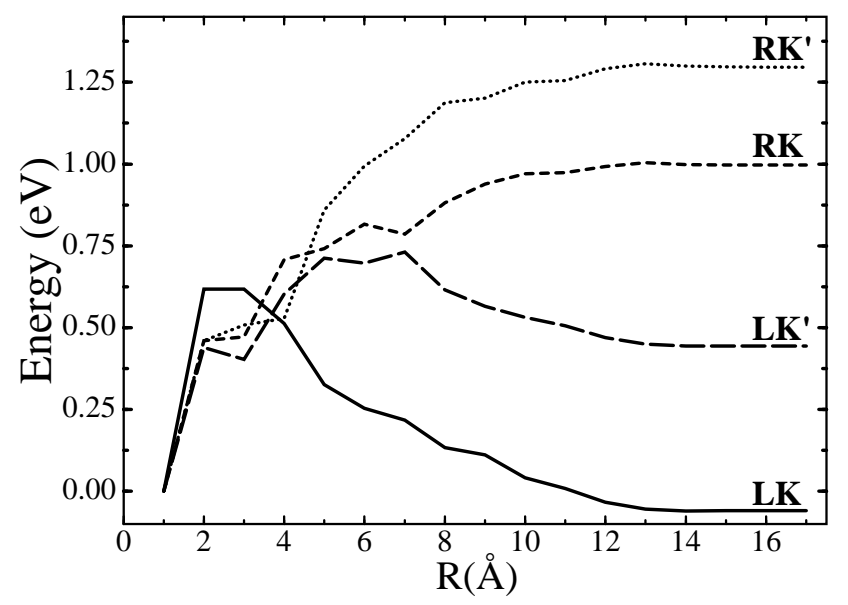

FIG. 5. Keating energy for $30^{\circ}$-partial kinks. Energy $E(R)$ is the sum over all atoms within a distance $R$ from the dislocation core. Corresponding core energy is subtracted to yield defect energies.

highest in energy. As we advance away from the core of the kinks, the energies only approach their final relative values at a distance of about $R=10.0 \AA$.

As in the case of the left kinks, a look at the saddlepoint configuration shows that the rather high migration barrier of $2.03 \mathrm{eV}$ for the right kinks is associated with the drastic bond distortions and malcoordination of atoms at the core. Note that this barrier is substantially higher than the 1.52 value we obtained for the left kinks, leading to a physical picture of "fast" and "slow" plasticity carriers for the $30^{\circ}$ partial dislocation. In our concluding section, we discuss this point further.

Here, it is worth pausing to compare our results with those in Ref. 13. Individual kink energies are not obtained in their work, since in all their calculations the supercells contained a double kink (two kinks, one of each family). Therefore, we cannot compare our kink energies directly with their results. In their procedure, what is computed are the relative energies of kinks within each kink family, assuming the LK and RK to have the same energy. A first aspect to be pointed out is that the above assumption of degeneracy between the LK and RK kinks is in sharp disagreement with our findings. In agreement with our work, they find an energy difference of $\sim 0.4 \mathrm{eV}$ between the two left kinks. On the other hand, while our results indicate that the two right kinks also differ by $\sim 0.4 \mathrm{eV}$, they find these two kinks to be almost degenerate, with energies differing by $0.07 \mathrm{eV}$ only. Our kink migration barriers are substantially higher, despite the fact that the associated saddle-point configurations seem to be very similar with those identified in their work. Below, our results will be seen to compare more favorably with experimental estimates of the kink barriers. 
TABLE II. Formation energy of defect complexes in the $30^{\circ}$ partial dislocation, in $\mathrm{eV}$. Two different states are considered for each complex (notation is explained in the text). Binding energy for the largest cell is indicated in the last column.

\begin{tabular}{lcccc}
\hline \hline & 756 atoms & 924 atoms & 1078 atoms & Binding energy \\
\hline $\mathrm{LC}(0)$ & 1.11 & 0.97 & 0.88 & 0.80 \\
$\mathrm{LC}(1)$ & 1.78 & 1.66 & 1.58 & - \\
$\mathrm{RC}(0)$ & 1.90 & 2.09 & 2.15 & 0.42 \\
$\mathrm{RC}(1)$ & 2.43 & 2.55 & 2.64 & - \\
\hline \hline
\end{tabular}

\section{PSD-kink complexes}

Kinks and PSDs can be considered as the fundamental types of excitations in the dislocation cores. Important structural features and modes of dislocation dynamics can also be associated with the complexes formed by these basic defect types. Moreover, since RDs such as the PSD are malcoordinated (thus acting as weak links in the reconstructed core), they may act as preferential sites for the nucleation of double kinks, as suggested by Heggie and Jones.1.25 Possibly, a PSD-kink complex could result from such a nucleation process, as the double kink expands and eventually dissociates into single kinks. Therefore, it is important to understand the structure and energetics of these complexes.

Here, we consider the energetics of the PSD-kink complexes. The important questions concern whether or not these complexes form bound states, as well as the associated binding energies and migration barriers. We considered each of the PSD-kink complexes in two configurations, as shown in Fig. 6. The left complex $(\mathrm{LC}=$ $\mathrm{LK}+\mathrm{PSD})$ is shown in the state of closest approach, $\mathrm{LC}(0)$, Fig. 6(a), in which the two constituents overlap and cannot be distinguished; and in an extended state, LC(1), Fig. 6(b), in which the PSD and the kink have been separated to adjacent positions. The corresponding right-complex cases $\mathrm{RC}(0)$ and $\mathrm{RC}(1)$ are shown in Fig. 6(c) and (d), respectively. In Table II we show our results for the energies of these four configurations, where it can be seen that the PSD binds strongly with both the left and the right kinks, in agreement with Ref. 13. Contrary to what is found in Ref. 13, our results indicate the LC to be more strongly bound than the RC. From the binding energies and the energies of these more extended configurations, we obtain a lower bound of 0.80 $\mathrm{eV}$ (LC) and 0.49 (RC) for the dissociation barrier of these bound states. Below, these results will be shown to be in sharp contrast with those for kink-RD complexes in the SP reconstruction of the $90^{\circ}$ partial dislocation, which are found to be unstable. Finally, we note that the energy of the LC is lower than that of the PSD, making the former the more likely site for unpaired electrons in the core of the $30^{\circ}$ partial. (a)

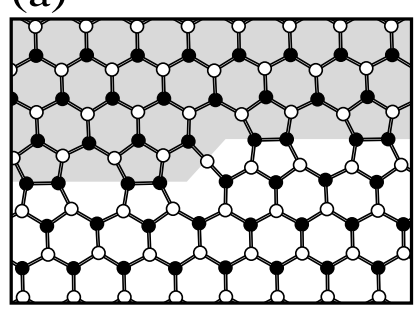

(c)

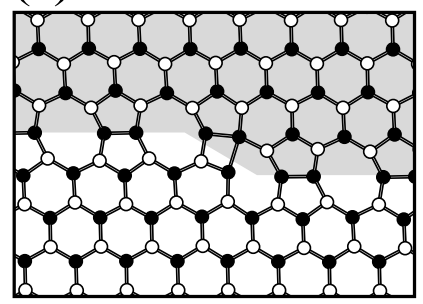

(b)

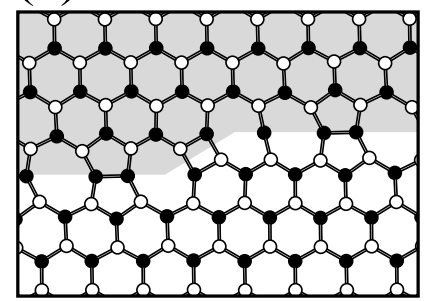

(d)

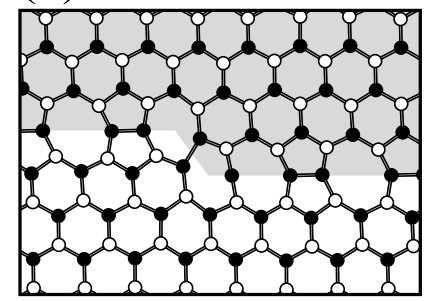

FIG. 6. Core structure of kink-PSD complexes in the $30^{\circ}$ partial. In each case, two states of the complex are considered, as explained in the text. (a) $\mathrm{LC}(0)=\mathrm{LK}+\mathrm{PSD}$ at zero separation. (b) $\mathrm{LC}(1)=\mathrm{LK}+\mathrm{PSD}$ one lattice period apart. (c) $\mathrm{RC}(0)=\mathrm{RK}+\mathrm{PSD}$ at zero separation. (d) $\mathrm{RC}(1)=\mathrm{RK}$ + PSD one lattice period apart.

\section{THE 90 ${ }^{\circ}$ PARTIAL DISLOCATION}

\section{A. Core reconstruction}

Considerable theoretical effort has been devoted to the study of the $90^{\circ}$ partial dislocation. $14,16,20,27 \mathrm{Ba}$ sically, two types of core reconstruction have been considered. These are the symmetric quasi-fivefold (QF) and the symmetry-breaking SP reconstructions shown in Fig. $7(\mathrm{a})$ and (b), respectively. Both preserve the original periodicity of the lattice along the dislocation direction. The latter structure has been found to be lower in energy. It was thus commonly assumed to be the ground state in $\mathrm{Si}$ and other semiconductors, and the bulk of studies of core excitations has relied upon this assumption. Recently, we proppsed an alternative solution for the ground-state in Si, 19 where a period-doubling symmetry-breaking structure, seen in Fig. 7(c), is shown to have lower energy than the SP one. As a consequence, the study of core excitations and the related issue of dislocation mobility have to be re-addressed. We are currently undertaking this task, and the results will be published elsewhere.

Nevertheless, we note that the DP structure is closely related to the SP one, being obtained by inserting alternating kinks in the core of the latter.19 Therefore, understanding the defect structure of the simpler SP core may prove useful to the study of the rather large number of core defects of the DP reconstruction. In this section, we summarize our main results for the core and related defects of the SP structure. 
(a)

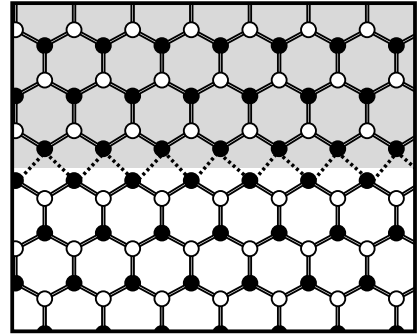

(c)

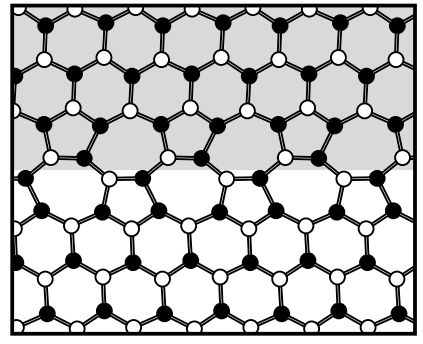

(b)

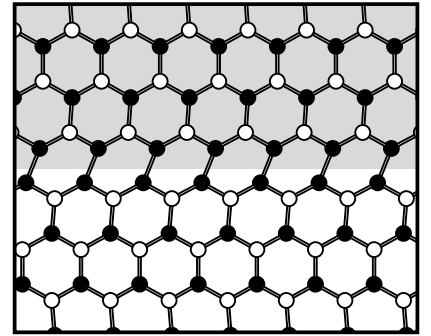

(d)

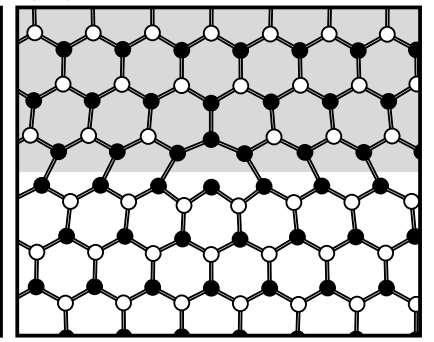

FIG. 7. Models for core reconstruction of the $90^{\circ}$ partial dislocation. (a) Symmetric QF reconstruction. (b) Symmetry-breaking SP structure. (c) Ground state symmetry-breaking DP structure. (d) Reconstruction defect or DSD in the SP core.

The SP core has two degenerate ground states, depending on the direction of the symmetry-breaking bonds. By convention, we denote the configuration in Fig. 7(b) as the "right" reconstruction, from which we can obtain the "left" state by applying the broken mirror operations [the ones that are unbroken in the QF core in Fig. 7(a)]. We find the SP core to be $0.18 \mathrm{eV} / \AA$ lower in energy than the $\mathrm{QF}$ gne. This result is in good agreement with previous TB16 $(0.18 \mathrm{eV})$ and LDA14 $(0.23 \mathrm{eV})$ works. In Ref. 14, it was found that symmetry breaking occurs spontaneously, a result that is confirmed by our model. In our calculations, the reconstructed bonds are stretched $3.0 \%$ with respect to the perfect crystal values (2.5\% in Ref. 14), and the minimum and maximum bond angles are $97^{\circ}$ and $135^{\circ}$, respectively $\left(96^{\circ}\right.$ and $138^{\circ}$ in Ref. 14). Core defects are considered next.

\section{B. Direction switching defect (DSD)}

Symmetry breaking in the SP core gives rise to a RD in which the direction of the bonds is switched, as shown in Fig. 7(d). We shall refer to this defect as a direction switching defect (DSD).28 Note that, like the $30^{\circ}$-partial PSD, this defect contains a dangling bond, which explains its formation energy of $1.45 \mathrm{eV}$. Our result is in reasonable agreement with the $1.2 \mathrm{eV}$ value obtained in the cluster calculations of Ref. 26. For the DSD motion, we computed an energy barrier of only $0.04 \mathrm{eV}$ for the propagation between two adjacent equilibrium positions. (a)

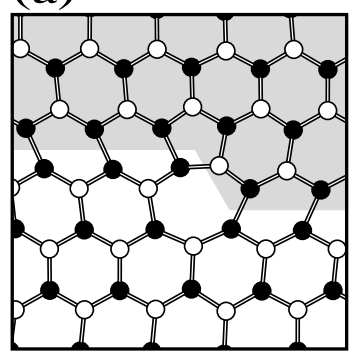

(c)

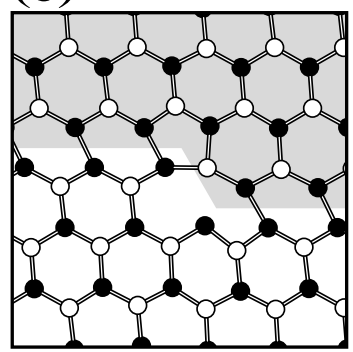

(b)

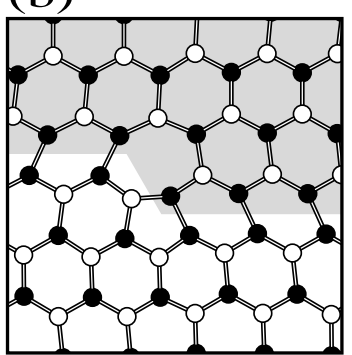

(d)

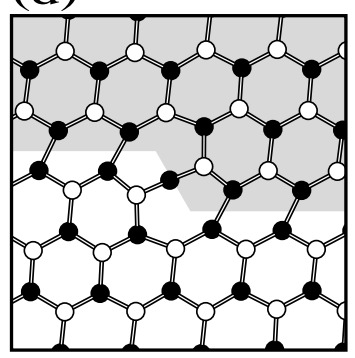

FIG. 8. Core structure of kinks and DSD-kink complexes in the SP core. See text for notation. (a) LR kink. (b) RL kink. (c) LL complex $=\mathrm{LR}+\mathrm{DSD}$. (d) RR complex $=\mathrm{LR}(\mathrm{RL})+$ DSD.

Given such a small barrier, the DSD is expected to be extremely mobile even at low temperatures. As a test, we performed a molecular dynamics simulation on a supercell having a pair of DSD defects, initially separated by $9.6 \AA$, on an otherwise defect-free partial dislocation. Remarkably, at a temperature of only $50 \mathrm{~K}$, recombination of the pair took place after only $1.3 \mathrm{ps}$. Unlike PSDs in the $30^{\circ}$ partial, such highly mobile DSDs do not bind strongly with kinks to form DSD-kink complexes, as explained below.

\section{Kinks}

It would be possible to define left (LK) and right (RK) kinks in the case of the $90^{\circ}$ partial, just as for the $30^{\circ}$ partial. However, in the $90^{\circ}$ case, each LK is directly related to a corresponding RK by application of a mirror symmetry. (This was not true for the $30^{\circ}$ partial, where the mirror symmetry was absent from the outset.) Thus, for the $90^{\circ}$ partial, we shall restrict the discussion to right kinks only. Moreover, we will now use the notation ' $L$ ' and ' $\mathrm{R}$ ' in a completely different way, namely, to denote the direction of the core reconstruction on either side of the kink. Referring to Fig. 8(a), the reconstruction will be said to tilt to the 'left' and to the 'right' on the left and right sides of the kink, respectively. Hence, we call this a left-right (LR) kink, the notation following accordingly for the other defects.

We compute the sum of the energies of the LR and RL kinks shown in Fig. 8(a) and (b), to be $0.24 \mathrm{eV}$ only. 
The RL and LR kinks are structurally quite similar; they would be related by a two-fold rotation axis normal to the plane of Fig. 8, if it were not for the fact that a stacking fault exists on one side but not the other. Thus we expect the energies of the two kinks to be similar, and assign the average energy of $0.12 \mathrm{eV}$ to each. The rather low formation energy can be seen as another indication of the DP core structure, since even individual kinks add little strain over that imposed by the SP core itself. In the formation of the DP core, this additional strain is more than compensated for by the attraction between the LR and RL kinks. In the present context, this result only shows that our previous results for kink energies in Ref. 15 were severely under-converged with respect to the dislocation interaction in the cell. We also computed an energy barrier of $1.62 \mathrm{eV}$ for the motion of the LR and $\mathrm{RL}$ kinks. As is the case for reconstructed kinks in the $30^{\circ}$ partial, such large energy barriers are associated with the existence of malcoordinated atoms and severe bond distortions at the core of the kink.

\section{DSD-kink complexes}

There are two additional kink-type defects associated with the SP reconstruction of the core. These are the RR and the LL defects, shown in Figs. $8(c)$ and (d). We prefer to regard these as complexes of a LR or a RL kink together with a DSD. Two LL complexes are possible (only one is shown in Fig. 8), and they share the same "quasi-symmetry" that the LR and RL kinks do, differing only by the position of the fivefold and dangling-bondcontaining rings with respect to the stacking fault. In contrast with complexes in the $30^{\circ}$ partial, these complexes appear to be either unstable or marginally stable against the emission of a DSD, as discussed in Ref. 15. The dissociation barrier, if present, is basically the DSD migration barrier, which indicates that these complexes should dissociate very easily at moderate temperatures. This was confirmed by a simulation performed at $300 \mathrm{~K}$, with a supercell containing a pair of RR complexes in each dislocation, separated by a distance of $34.6 \AA$. On the time scale of $1 \mathrm{ps}$, one of the kink complexes undergoes the DSD-emission reaction $\mathrm{RR} \rightarrow \mathrm{RL}+\mathrm{DSD}$, with the DSD propagating rather easily towards the other RR complex, where a DSD $+\mathrm{RR} \rightarrow \mathrm{LR}$ process takes place. Overall, a dislocation containing a pair of $\mathrm{RR}$ complexes relaxes into one containing alternating RL and LR kinks, by means of DSD emission (absorption) and propagation.

\section{COMPARISON WITH EXPERIMENTAL RESULTS}

In Table III we summarize our results for the formation energies and migration barriers of kinks in the $90^{\circ}$ and $30^{\circ}$ partial dislocations. For the $30^{\circ}$ partial, of
TABLE III. Formation energy and migration barriers of dislocation kinks in $\mathrm{Si}$, in eV. Range of available experimental estimates of is included. For comparison, results from Ref. 13 are also include.

\begin{tabular}{|c|c|c|c|}
\hline Dislocation & Kink type & Formation energy & Migration barrier \\
\hline $30^{\circ}$ & LK & $0.35\left(0.82^{\mathrm{a}}\right)$ & $1.53\left(0.82^{\mathrm{a}}\right)$ \\
\hline $30^{\circ}$ & RK & $1.24\left(0.82^{\mathrm{a}}\right)$ & $2.10\left(0.74^{\mathrm{a}}\right)$ \\
\hline $90^{\circ}$ & LR & 0.12 & 1.62 \\
\hline $90^{\circ}$ & $\mathrm{RL}$ & 0.12 & 1.62 \\
\hline Experiment & & $0.4-0.7$ & $1.2-1.8$ \\
\hline
\end{tabular}

${ }^{\text {a From Ref. } 13}$

the two equilibrium states of each kink $\left[\left(\mathrm{LK}, \mathrm{LK}^{\prime}\right)\right.$ and $\left.\left(\mathrm{RK}, \mathrm{RK}^{\prime}\right)\right]$, one is to be regarded as an intermediate metastable state in the propagation of the kink, given the substantial difference in formation energy between the two states. Only the state with the lower formation energy will determine the kink concentration in each case (this lower formation energy is the number included in Table III). For comparison, results from Ref. 13 are also included, as are the ranges of experimental results for both quantities, obtained from different techniques.t. We observe that, for the $30^{\circ}$ partial, our values are in excellent agreement with the experimental ones.

The interpretation of these experiments is done according to the theory of Hirth and Lothe. In this theory, the dislocation velocity is given by

$$
v_{d} \propto 2 \times \exp \left[-\frac{1}{k T}\left(U_{k}+W_{m}\right)\right],
$$

where $U_{k}$ is the kink formation energy and $W_{m}$ is the kink migration barrier. This equation is written under the assumption that the two kinks that result from the nucleation of a stable double kink (a kink-antikink pair) are equivalent. This assumption does not hold for the $30^{\circ}$ partial, where the left and right kinks are intrinsically different. The more general form

$$
\begin{aligned}
v_{d} & \propto \exp \left[-\frac{1}{2 k T}\left(U_{L K}+U_{R K}\right)\right] \\
& \times\left[\exp \left(-\frac{W_{m}^{L K}}{k T}\right)+\exp \left(-\frac{W_{m}^{R K}}{k T}\right)\right],
\end{aligned}
$$

must be used. We note that the quantity of interest in the first activated term is the average formation energy of the two kink species. The second term is derived from the kink velocities, and therefore the relative velocity appears in the generalized form. In the $30^{\circ}$ partial this term is dominated by the velocity of the left kinks (fast carriers), given the much higher migration barrier of the right kinks (slow carriers). We should point out that the average formation energy of the kink-antikink pairs in Table III falls within the range of the experimental numbers, for the $30^{\circ}$ partial. As we mentioned in the introduction another theory of dislocation glide has been proposed,10.11 in which the motion is controlled by the 
pinning of kinks by strong obstacles along the dislocation line, and the kink migration barriers are not rate controlling. Despite the fact that our work does not address such pinning mechanisms, and thus cannot clearly decide between these two theories, our results are certainly consistent with the HL interpretation.

Strictly speaking, our comparison is only valid for the $30^{\circ}$ partial, since we did not consider the true ground state for the $90^{\circ}$ partial. In the latter case, the excellent agreement we obtain for the kink barriers appears to be fortuitous. Nevertheless, our results are qualitatively consistent with the experimental images in Ref. 7, which show a higher concentration of kinks in the $90^{\circ}$ partial. In Table III, we see that kink energies are lower in this dislocation, as compared to the $30^{\circ}$ partial. Obviously, this is only plausible to the extent that this general trend of lower kink energies carries over to the ground-state DP structure of the $90^{\circ}$ partial.

\section{CONCLUSIONS}

In this work, an extensive study of the core reconstruction and structural excitations in the cores of both the $30^{\circ}$ and the $90^{\circ}$ partial (in its $\mathrm{SP}$ reconstruction) in $\mathrm{Si}$, was presented. For both partials, we find the core to undergo strong bond reconstruction, restoring the fourfold coordination of the core atoms. The reconstructed bonds are stretched by only $\sim 3 \%$ with respect to bulk values, and the core energies are mostly associated with the bond angle distortions present in the reconstructed cores.

In the case of the SP structure of the $90^{\circ}$ partial, the $\mathrm{RD}$ (or DSD) is associated with a switch of direction of the reconstructed bonds, and is found to be highly mobile. Kink-DSD complexes are found to be only marginally stable against emission of a DSD, a reaction that is observed to proceed rather quickly in our simulations at room temperature. The LR and RL kinks have very low formation energy, indicating that they introduce little additional on the SP core, a result which is consistent with the lower energy of the DP core, as proposed in Ref. 19.

For the $30^{\circ}$ partial, two kink species (RK and LK) are identified, and the RK's are found to have higher formation energies that the LK ones. This is explained by the medium-range behavior of the associated strains. The $\mathrm{RD}$ (or PSD) is related to a phase-switching of the core reconstruction, and binds strongly with kinks to form PSD-kink complexes. These are the more likely sites for unpaired electrons in the $30^{\circ}$ partial core. The results for this particular dislocation can be directly compared with experiment, and we find good agreement between our calculated values for the kink formation energies and migration barriers and the experimental results.

\section{ACKNOWLEDGMENTS}

Partial support was provided by the DoD Software Initiative. J.B. and D.V. acknowledge support from NSF Grants DMR-91-15342 and DMR-96-13648.

${ }^{1}$ P. B. Hirsch, Mater. Sci. Tech. 1, 666 (1985).

${ }^{2}$ M. S. Duesbery and G. Y. Richardson, Crit. Rev. Solid State Mater. Sci. 17, 1 (1991).

${ }^{3}$ H. Alexander and H. Teichler, in Materials Science and Technology, edited by R. W. Cahn, P. Hassen, and E. J. Kramer (VCH Weiheim, Cambridge, 1993), Vol. 4, p. 249.

${ }^{4}$ H. Gottschalk et al., Phys. Stat. Sol. (a) 138, 547 (1993).

${ }^{5}$ B. Ya. Farber, Yu. L. Iunin, and V. I. Nikitenko, Phys. Stat. Sol. (a) 97, 469 (1986); B. Ya. Farber et al., ibid. 138, 557 (1993).

${ }^{6}$ V. I. Nikitenko, B. Ya. Farber, and Yu. L. Iunin, Sov. Phys. JETP 66, 738 (1987).

${ }^{7}$ H. R. Kolar, J. C. H. Spence, and H. Alexander, Phys. Rev. Lett. 77, 4031 (1996).

${ }^{8}$ J. P. Hirth and J. Lothe, Theory of Dislocations, (Wiley, New York, 1962), p. 531.

${ }^{9}$ K. Maeda and Y. Yamashita, in International Symposium on the Structure and Properties of Dislocations in Semiconductors, edited by S. G. Roberts, D. B. Holt, and P. R. Wilshaw (IOP Publishing Ltd, , Bristol, 1989), p. 269.

${ }^{10}$ V. Celli, M. Kabler, T. Ninomiya, and R. Thomson, Phys. Rev. 131, 58 (1963).

${ }^{11}$ V. Rubin and A. Orlov, Sov. Phys. Solid State 11, 2635 (1970).

${ }^{12}$ F. H. Stillinger and T. A. Weber, Phys. Rev. A 25, 978 (1985).

${ }^{13}$ V. V. Bulatov, S. Yip, and A. S. Argon, Philos. Mag. A 72, 453 (1995).

${ }^{14}$ J. R. K. Bigger et al., Phys. Rev. Lett. 69, 2224 (1992).

${ }^{15}$ R. W. Nunes, J. Bennetto, and D. Vanderbilt, Phys. Rev. Lett. 77, 15417 (1996).

${ }^{16}$ L. B. Hansen et al., Phys. Rev. Lett. 75, 4444 (1995).

17 T. A. Arias and J. D. Joannopoulos, Phys. Rev. Lett. 73, 680 (1994).

${ }^{18}$ Y. M. Huang, J. C. Spence, and O. F. Sankey, Phys. Rev. Lett. 74, 3392 (1995).

19 J. Bennetto, R. W. Nunes, and D. Vanderbilt, Phys. Rev. Lett. 79, 245 (1997).

20 J. R. Chelikowsky, J. C. H. Spence, Phys. Rev. B 30, 694 (1984).

${ }^{21}$ S. Marklund, J. Phys. (Paris), Colloq. 44, C4-25 (1983).

22 S. Marklund and Y.-L. Wang, Solid State Commun. 91, 301 (1994).

${ }^{23}$ R. Jones, Philos. Mag. B 42, 213 (1980).

${ }^{24}$ R. Jones et al, Phys. Status Solidi 138, 369 (1993).

${ }^{25}$ M. I. Heggie and R. Jones, Philos. Mag. B 48, 365 (1983); 48379 (1983). 
${ }^{26}$ M. I. Heggie, R. Jones, and A. Umerski, Phys. Stat. Sol 138, 383 (1993).

${ }^{27}$ S. Öberg, P. K. Sitch, R. Jones, and M. I. Heggie, Phys. Rev. B 51, 13138 (1995).

${ }^{28}$ Reconstruction defects in the core of the $30^{\circ}$ and $90^{\circ}$ partials have commonly been termed as solitons, but variants such as antiphase defect have also been used. In this paper, we shall refer to these as phase-switching defect, in the case of the $30^{\circ}$, and direction-switching defect in the case of the $90^{\circ}$ partial, as explained in the text.

${ }^{29}$ I. Kwon et al., Phys. Rev. B 49, 7242 (1994).

${ }^{30}$ X.-P. Li, R. W. Nunes, and D. Vanderbilt, Phys. Rev. B 47, 10891 (1993).

${ }^{31}$ P. N. Keating, Phys. Rev. 145, 637 (1966).

${ }^{32}$ The reaction path is the path joining the two minima in configuration space, along which the gradient is always parallel to the path. Physically, it is the minimal-action path that the system would follow to reach a minimum once released from the saddle-point configuration.

33 J. E. Northrup, et al., Phys. Rev. B 24, 4623 (1981).

${ }^{34}$ J. R. Chelikowsky, Phys. Rev. Lett 49, 1569 (1982).

${ }^{35}$ G.-X. Qian and D. J. Chadi, J. Vac. Sci. Technol. B 4, 1079 (86). 